\title{
LOS CICLOS CIUDAD CINE, CIUDAD ESCRITA Y CIUDAD DIBUJADA. \\ ESTRATEGIAS DE BORDE PARA EL DESARROLLO DE \\ PROCESOS DE APRENDIZAJE SOBRE LA CIUDAD Y EL ESPACIO PÚBLICO
}

\section{FILM CITY, WRITTEN CITY AND PICTURE CITY SERIES. BORDER STRATEGIES TO DEVELOP PROCESSES OF LEARNING ABOUT CITY AND PUBLIC SPACE}

ALCALÁ, Laura I.

Arquitecta UNNE. Doctora por la Universidad Politécnica de Cataluña en el Programa Urbanismo. JTP Ded. Exc. Arquitectura II. UPC. FAU. UNNE. Investigadora Adjunta CONICET y del Instituto de Investigación y Desarrollo en Vivienda. FAU. UNNE. Directora PI SGCYT C001/08. Espacio Público en el Gran Resistencia. Diagnóstico y Propuesta para el mejoramiento integral del sistema de calles (2009-2012).

Palabras Clave: Procesos de aprendizaje - Estrategias de borde - Ciudad y espacio Keywords: Learning Processes - Border Strategies - City and Space.

\section{RESUMEN}

El trabajo expone resultados y reflexiones de una experiencia que se desarrolló con la modalidad de tres ciclos de debate sobre la ciudad y el espacio público: CIUDAD CINE; CIUDAD ESCRITA y CIUDAD DIBUJADA, en la Facultad de Arquitectura y Urbanismo de la UNNE durante los años 2010, 2011 y 2012, en el marco de las actividades complementarias de articulación con la docencia en el Taller de Arquitectura, previstas en el Proyecto de Investigación "Espacio Público en el Gran Resistencia. Diagnóstico y Propuesta para el mejoramiento integral del sistema de calles", acreditado por la SGCYT y desarrollado entre 2009 y 2012.

\section{ABSTRACT}

This work shows the results of and reflections on an undertaking which took place in the form of three discussion series about city and public space: FILM CITY, WRITTEN CITY and PICTURE CITY at the Architecture and Urbanism College of UNNE in the years 2010, 2011 and 2012 as a part of the complementary activities of collaboration with teachers at the Architecture Workshop outlined in the Research Project "Public Space in Greater Resistencia. Assessment and Proposals for the Complete Improvement of Streets", validated by SGCYT and carried out between 2009 and 2012. 
Participaron alumnos de $1 .^{\circ}$ a $6 .^{\circ}$ año, incluso alumnos del colegio secundario, y docentes de las áreas de Diseño, de las Ciencias Sociales, y del Entorno y del Ambiente, además de artistas plásticos y de un escritor.

\section{INTRODUCCIÓN}

Este trabajo se plantea como objetivo analizar una experiencia de aprendizaje (1) sobre la ciudad y el espacio público que se propuso abordar integralmente la naturaleza multidimensional del objeto de estudio y apostar por la construcción colectiva del conocimiento (2), mediante la generación de una estrategia de borde (3), que permitiera integrar contenidos de distintas asignaturas de la Carrera de Arquitectura y distintos saberes (4), utilizando el arte como herramienta didáctica (5).

1. Aprendizaje en el sentido planteado por Edith Litwin, según el cual enseñanza-aprendizaje no son parte de un proceso lineal unidireccional sino un proceso de desarrollo espiralado en el que los docentes investigadores también son sujetos del aprendizaje. (LITWIN, 2011)

2. Construcción colectiva del conocimiento, a partir de la conformación de una comunidad de aprendizaje ${ }^{1}$ inclusiva de integrantes de los distintos claustros (profesores, auxiliares de la docencia y estudiantes) y de sujetos que no forman parte de la comunidad académica universitaria (en este caso particular, artistas), a partir del aprovechamiento de sus distintos saberes, puestos en un plano de valoración equitativo y basado en el reconocimiento y el respeto mutuo (FREIRE, 1993). Ello supone que la visión sobre la ciudad de un investigador no es ni mejor ni más imperfecta que la de un artista, sino que son, cada una, miradas parciales que pueden complementarse en pos de una comprensión más holística y menos fragmen-

[1] Si bien el concepto de comunidad de aprendizaje es mucho más amplio y supone un proyecto educativo institucional, en general aplicado a comunidades escolares como lo plantean FLECHA GARCIA y PUIGVERT, lo utilizamos en este caso para dar cuenta de la intencionada construcción de un espacio interactivo entre un grupo de personas diferentes (de distintas edades y saberes), con el fin de aprender en común, utilizando herramientas comunes, en un mismo entorno y apostando por el aprendizaje dialógico para el cual deben, darse entre otros factores; el diálogo igualitario e inclusivo, donde las diferentes aportaciones son consideradas en función de la validez de los argumentos y no desde el posicionamiento jerárquico donde un profesor determina los contenidos de lo que es necesario aprender; un aprendizaje solidario en el cual cada uno es educador y educando y donde se busca producir una construcción colectiva de sentido. tada que nos aproxime de un modo más abierto a una realidad compleja.

3. Estrategia de borde. Si bien aquí partimos también de la noción desarrollada por Edith Litwin², que no hace referencia a un tipo de contenidos periférico sino a una búsqueda didáctica por desarrollar de una manera novedosa e interesante un tema central; en este trabajo también queremos referirnos con ello a la posición del espacio de aprendizaje, ubicado por fuera de la currícula de la Carrera de Arquitectura. Aun cuando esta estrategia se desarrolló en el marco de un proyecto de investigación acreditado por la SGCYT-UNNE y en estrecha articulación con la programación anual de la asignatura -Arquitectura III de la Unidad Pedagógica C (UPC)-, se trató de un espacio extraaúlico abierto, que no formó parte de ninguna asignatura en particular y que no se ciñó a un nivel determinado de la carrera, ni a un área particular del conocimiento. Este espacio se configuró en torno a tres ciclos de Debate sobre la Ciudad y el Espacio Público que fueron declarados oportunamente de Interés Institucional por el Consejo Directivo de la FAU, pero que no formaron parte de cursos normados y acreditables.

4. Participaron alumnos de $1 .^{\circ}$ a $6 .^{\circ}$ año, incluso alumnos del colegio secundario, y docentes de las áreas de Diseño, de las Ciencias Sociales, y del Entorno y del Ambiente, además de artistas plásticos y de un escritor.

5. Los ciclos tuvieron un mes de duración cada uno, con un encuentro semanal en cada caso, por las tardes, y se repitieron durante tres años. Cada uno se configuró a partir de la selección de un tipo

[2] Para Edith Litwin, una estrategia de borde no tiene que ver con conceptos periféricos o marginales o de borde curricular sino con "el desafío didáctico de hacer atractiva la enseñanza de contenidos centrales mediante el desarrollo de prácticas originales o divertidas, la introducción del humor o la búsqueda de cursos de acción novedosos". (LITWIN, 2011: 40). 
El espacio público del AMGR presentaba importantes diferencias negativas entre distintos sectores centrales y periféricos, se encontraba en franco deterioro, conflictividad y siniestralidad creciente, etc. (ALCALÁ, 2010).

de expresión artística, utilizándola como herramienta didáctica para proponer distintos temas de reflexión en torno a la ciudad y el espacio público (EP). Cada jornada tuvo una instancia expositiva y otra de debate. En el primero, Ciudad Cine, se trabajó con el cine; en el segundo Ciudad Escrita, con la literatura y, en el tercero, Ciudad Dibujada, con el dibujo.

\section{ORIGEN Y CARACTERÍSTICAS DE LA EXPERIENCIA}

Para situar el origen de la experiencia es preciso retroceder al año 2008 cuando se formuló el proyecto de investigación "Espacio Público en el Gran Resistencia. Diagnóstico y Propuesta para el mejoramiento integral del sistema de calles"(PI EP) ${ }^{3}$. En aquel momento, razones de distinto tipo: el diagnóstico preliminar de la situación del espacio público en el Área Metropolitana del Gran Resistencia, AMGR (1); el espacio absolutamente marginal que tenía su abordaje dentro de la FAU (2); la casi inexistente práctica investigativa dentro del Área de Diseño (3), y los alentadores resultados de una experiencia de diseño urbano iniciada en el año 2006 dentro de los contenidos de la Asignatura Arquitectura III de la UPC (4), constituyeron razones que nos llevaron a considerar la necesidad, por un lado, y la oportunidad por el otro, de formular un proyecto investigación radicado en el Departamento de Diseño Arquitectónico y Urbano que permitiera abordar de manera integral el estudio del EP del Gran Resistencia para avanzar

[3] PI SGCYT C001/08. Directora: Laura Alcalá. Integrantes: María Dora Foulkes, Regina Pérez, Marina Scornik, Paula Valdés, Aníbal Bennato, Walter González y del que también formaron parte Carolina Brito y Juan Sorochin (alumnos ya recibidos.) en la formulación de patrones de diseño para su mejoramiento integral.

1. El espacio público del AMGR presentaba importantes diferencias negativas entre distintos sectores centrales y periféricos, se encontraba en franco deterioro, conflictividad y siniestralidad creciente, etc. (ALCALÁ, 2010). Si desde entonces ahora puede decirse que el reconocimiento de la importancia de repensar las condiciones del EP se ha extendido más, tanto a nivel académico como de los municipios que conforman este territorio metropolitano y se han introducido algunos cambios, los resultados poco alentadores de las condiciones actuales del EP del AMGR avalan con mayor sustento la pertinencia en aquel momento de encarar su diagnóstico y también la de avanzar en el presente en la propuesta de mejoras.

2. La ciudad y en menor medida, el espacio público, son abordados por distintas asignaturas de la Carrera de Arquitectura de la FAU-UNNE: desde la historia, el desarrollo urbano, la planificación, la morfología o el paisaje, desde el sesgo temático que imprime cada una de ellas y desde los objetivos y contenidos definidos en sus programas. En menor grado y solo tangencialmente, también forman parte, en tanto objeto de diseño, en los talleres de Arquitectura. En este caso, decimos "tangencialmente", porque el trabajo en los talleres está fundamentalmente orientado al diseño de objetos arquitectónicos, y en general los pocos casos en que llega a producirse diseño urbano se circunscriben al diseño singular de un parque o una plaza u obedecen a la voluntaria adopción como tema de desarrollo en los proyectos finales de carrera (PFC). Esta ausencia de práctica en el diseño urbano, aunque cuestionable desde nuestro punto de vista, no será discutida en este trabajo sino simplemente tomada como dato de una realidad que responde indudablemente al diseño de una currícula que expresa los intereses de la comunidad académica que la concibió y que inevitablemente también 


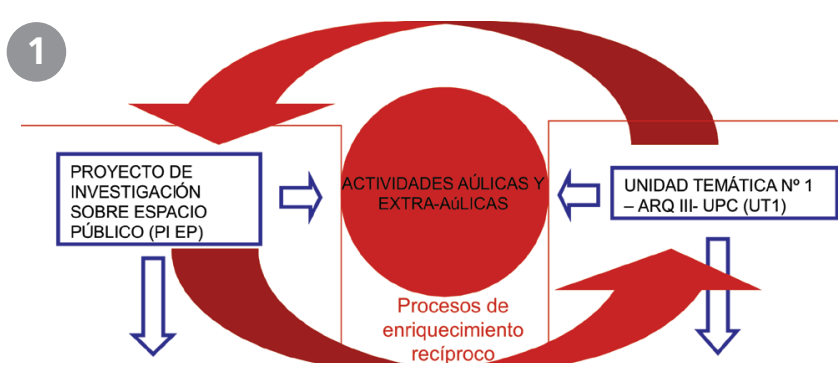

se refleja en las ciudades donde actúa la mayor parte de los egresados de esta casa de estudios ${ }^{4}$. 3. Si en el contexto de nuestra Universidad, la práctica investigativa dentro de la Facultad de Arquitectura y Urbanismo es menos frecuente que en otras Facultades, la investigación en diseño es más infrecuente aún y tenía contados ejemplos cuando propusimos hacerlo en el año 2008.

4. Desde el año 2006, en la asignatura Arquitectura III de la UPC ${ }^{5}$, se puso en práctica una primera unidad temática (UT.1) destinada a conocer y comprender la ciudad donde se realiza posteriormente el ejercicio de diseño arquitectónico, y destinada a realizar un ejercicio de diseño urbano sobre un eje urbano, previamente analizado y diagnosticado por los mismos estudiantes, con el objeto de desarrollar una propuesta de mejoramiento integral del EP6. De este modo, más allá de los objetivos específicos de la investigación ${ }^{7}$, el proyecto se formuló con propósitos complementarios: por un lado, servir de manera directa a la actividad docente que Ilevaban adelante en el taller de Arquitectura III-UPC parte de los integrantes del equipo de investigación del PI EP y, por el otro, posicionar la ciudad y el espacio público como temas centrales de interés dentro de la FAU. Se trataba del inicio de un campo nuevo de abordaje, para un equipo que se acababa de conformar, dado que no existían antecedentes de investigación conjunta entre los integrantes del proyecto de investigación, lo que exigió poner especial énfasis en la construcción paulatina de un marco teórico común que pasara de ser la sumatoria de conocimientos y puntos de vistas individuales -construidos en la trayectoria personal de cada uno-, como vía indispensable para el acercamiento colectivo al objeto de estudio.

[4 ] En ALCALÁ, 2010 op. cit. se exponen distintos ejemplos de desconsideración del diseño urbano y de EP en las acciones habitacionales, viales, etc.

[5 ] La Cátedra está integrada por la Profesora Titular María Dora Foulkes, los JTP Laura Alcalá, Carlos

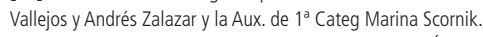

[6] Puede leerse sobre esta unidad temática en ALCALÁ, L.; FOULKES, M. D.; SCORNIK, M. (2005).

[7] Objetivos fueron expuestos en otros trabajos como ALCALÁ, L.; FOULKES, M. D.; SCORNIK, M. (2009).

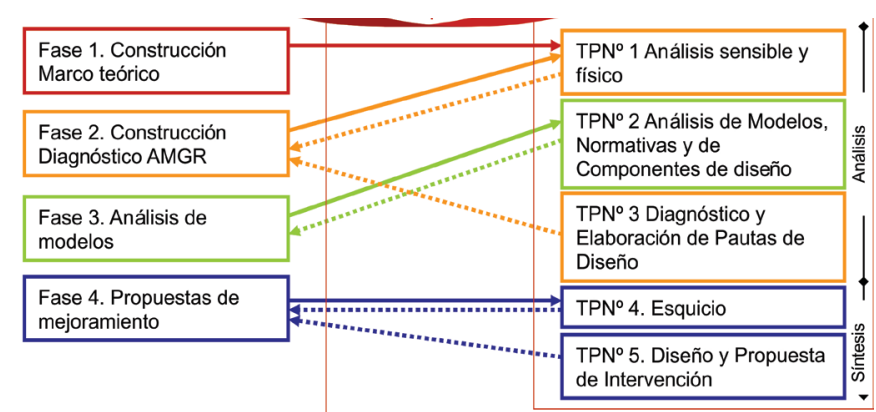

Desde su formulación, se hicieron expresos algunos criterios sostenidos por la dirección del proyecto que con el desarrollo de la experiencia fueron siendo fortalecidos por el equipo de trabajo y que hacen a los conceptos referidos previamente: la apuesta por la integración de saberes; por la construcción de espacios alternativos al aula y al gabinete de investigación; la apuesta por el intercambio con el medio y la sociedad; la necesidad de explorar recursos alternativos para abordar la naturaleza polifacética del EP, para intentar superar la visión estrictamente disciplinar y para llegar a comprender integralmente el objeto de estudio, lo cual era considerado un paso indispensable para poder proponer actuar sobre él. Nos referimos aquí a la necesidad de pasar del saber al comprender que plantea Manfred Max Neef (2003)

De esta manera, la experiencia que aquí se analiza puede situarse dentro de un conjunto de actividades extra-áulicas planteadas en el PI EP y en la programación anual de la asignatura Arquitectura III- UPC de los años 2010, 2011, 2012. Antes de llegar a ellas, haremos una breve referencia al organigrama general dentro del que estas aparecen situadas. (Fig. 1).

\section{Proyecto de investigación (PI EP)}

Se trata de un proyecto organizado en 4 fases de trabajo: Fase 1, de Construcción del marco teórico, en especial sobre el significado y la función de la ciudad y el espacio público; Fase 2, de Construcción del diagnóstico sobre la situación del espacio público del Área Metropolitana del Gran Resistencia (AMGR) desde una perspectiva multidimensional; Fase 3, de Análisis de Modelos de intervención en situaciones urbanas semejantes y Fase 4, de Propuesta de mejoramiento integral del sistema de calles del AMGR.

\section{Unidad Temática I de la Asignatura Arquitectura III-UPC}

Se trata de la primera UT que abordan los alunos 
Como parte de la articulación entre docencia e investigación se desarrollaron además una serie de actividades extra-áulicas. Este tipo de actividades puede clasificarse de acuerdo a dos orígenes

de taller de $3 .^{\text {er }}$ año, organizada en cinco trabajos prácticos. Los tres primeros correspondientes a la etapa de análisis del proceso de diseño urbano en un sector de la ciudad que tiene como eje central a alguna de las principales avenidas o calles de la ciudad de Resistencia: TPN. ${ }^{\circ} 1$, Análisis sensible y físico; TPN. ${ }^{\circ} 2$, Análisis de modelos, de la normativa vigente y de componentes de diseño urbano; TPN. ${ }^{\circ}$ 3, Diagnóstico y pautas de diseño. Los dos siguientes, forman parte de la etapa de síntesis. EI TPN. ${ }^{\circ} 4$, consiste en un Esquicio y el TPN. ${ }^{\circ} 5$ en una Propuesta de intervención a nivel de partido avanzado.

\section{Procesos de enriquecimiento recíproco}

Los avances de la Fase 1 del PI EP aportaron contenidos teóricos para presentar el tema de diseño y su problemática en el taller, para encauzar la observación y el análisis del sector urbano para intervenir (TPN. ${ }^{\circ}$ 1) así como para fundamentar determinados criterios de diseño en relación con la movilidad, la accesibilidad sin barreras, las principales características de un EP inclusivo y dignificante para el ciudadano, etc.

En este caso, se proveyó cada año a los estudiantes de herramientas de observación y análisis, así como de parámetros de referencias para la construcción del diagnóstico. Los avances de la Fase 2 aportaron contenidos para situar el sector de intervención y el problema de abordaje en el contexto metropolitano. En este caso, las clases teórico-prácticas del taller brindaron una aproximación al territorio urbano de trabajo, dado que hasta el momento, la gran mayoría de los estudiantes no lo habían hecho en las demás asignaturas. La producción diagnóstica que desarrollaron los estudiantes en los sectores definidos por la cátedra contribuyeron, en sentido inverso, a profundizar y perfeccionar el diagnóstico del equipo de investigación. Los avances de la Fase 3 permitieron contar con ejemplificaciones que ayudaron a ilustrar y explicar alternativas de diseño para situaciones semejantes a las aborda- das en el taller. De la misma manera y en sentido inverso, los análisis de modelos realizados por los alumnos contribuyeron a enriquecer y a perfeccionar la base de datos disponibles sobre operaciones urbanas de espacio público. Los resultados de la Fase 4 aportaron criterios de diseño, mientras que la propuesta de diseño de los equipos de estudiantes permitieron verificar hipótesis del equipo de investigación y ampliar perspectivas de diseño para la ciudad de estudio ${ }^{8}$.

La existencia de fases en el proyecto de investigación y no de etapas supuso el desarrollo casi en paralelo de cada una a lo largo de los cuatro años de duración del proyecto, reconociendo en ello la idea de proceso abierto y en construcción permanente. La relación con la actividad docente supuso en este contexto un compromiso de ajuste, verificación y reformulación anual que evitó quedar en una meseta en el desarrollo de cada fase del proyecto, por un lado, y en el estancamiento de los contenidos de la UT, por el otro.

\section{Actividades extra-áulicas}

Como parte de la articulación entre docencia e investigación se desarrollaron además una serie de actividades extra-áulicas. Este tipo de actividades puede clasificarse de acuerdo a dos orígenes: cuando surgieron como parte de la programación anual de la Cátedra y cuando surgieron del PI EP. Tuvieron en común que en ellas el proceso de aprendizaje se vio enriquecido por la incorporación de otros sujetos académicos y no académicos en su desarrollo, y que el espacio de aprendizaje se trasladó a la ciudad o a algún otro espacio que no fue el aula-taller.

Entre las que surgieron de la actividad docente se pueden citar: a) recorridos por el sector de estudio; visitas a ejemplos urbanos en las ciudades de Resistencia y Corrientes o bien de otras ciudades formando parte en estos casos de viajes de estudio. En

[8 ] Esta interrelación fue analizada con mayor profundidad en ALCALÁ, L.; FOULKES, M. D.; SCORNIK, M. (2009). 
todos los casos se trató de recorridos compartidos y comentados entre docentes, estudiantes y en lo posible por los profesionales que tuvieron participación en el diseño o transformación del sector visitado; b) charlas de profesionales invitados por la Cátedra para comentar sus proyectos de intervención; c) charlas de técnicos municipales invitados para explicar la política seguida por el municipio y los proyectos planificados en relación con el transporte público el mejoramiento urbano, etc.; d) charlas de profesionales de otras disciplinas invitados a transferir conocimientos específicos sobre, por ejemplo, arbolado urbano, comercio, etc. Entre las actividades que surgieron del PI EP se pueden citar: a) la organización de viajes de estudio que incluyeron el intercambio con técnicos, por lo general de los municipios de las ciudades visitadas (por ejemplo, Formosa y Posadas) y b) la organización de seminarios y ciclos como los que aquí se analizarán en mayor profundidad.

\section{UNA ESTRATEGIA DE BORDE PARA EL DESARROLLO DE PROCESOS DE APRENDIZAJE}

La experiencia que se analiza abarcó el desarrollo de tres ciclos que se caracterizaron por:

- Ser de participación obligatoria para los cursantes de Arquitectura III-UPC, pero de participación voluntaria para el resto de los estudiantes de la Carrera de Arquitectura.

- Ser gratuitos y estar abiertos a la comunidad en general.

- Estar planteados, cada uno, en torno a un tipo de expresiones artísticas, utilizándolas como herramientas o dispositivos didácticos para generar reflexiones o discusiones en distintos aspectos y temas relativos a la ciudad y el espacio público.

- Tener cada jornada de los tres ciclos un formato semejante: una instancia expositiva y otra de debate. En el caso de Ciudad Cine, la primera instancia consistió en la presentación de un film a cargo de un docente de las Cátedras con las que se articuló esta actividad ${ }^{9}$ y luego la proyección de la película. El mismo docente se encargó de la coordinación del debate posterior. En los otros ciclos, la primera instancia estuvo a cargo de uno, dos y hasta tres expositores invitados, y luego el debate fue coordinado por algún integrante del PI EP.

- Contar entre los expositores invitados con distintos docentes de la Carrera de Arquitectura de asignaturas teóricas y del taller, con alumnos y con artistas. El listado de los participantes es una demostración de la intencionada diversidad de los expositores (ver cuadro síntesis).

-Contar con un programa predefinido por el equipo de investigación que luego se configuró de manera definitiva con aportes y sugerencias propuestas por docentes amigos entusiastas de los ciclos. En el caso particular de Ciudad Cine, cada una de las cuatro cátedras con las que se articuló la actividad estuvo encargada de proponer y presentar un film. - Invitar a los expositores a que desarrollen un tema específico a partir de la idea general del programa, sin tener en cuenta sus temas habituales de enseñanza e investigación, sino más bien sus hobbies, alguna relación previa con la propuesta temática o bien de acuerdo con su perfil. En todos los casos exigió por parte de ellos un trabajo específico de investigación para poder desarrollarlo. - Desarrollarse cada jornada los miércoles (día de taller de $2 .^{\circ}$ a $6 .^{\circ}$ año) de cada mes de mayo a partir de las 18 hs, en general en el Salón Auditorio de la FAU.

- Contar con un sistema de comunicación que, tanto en el Ciclo Ciudad Cine como en Ciudad Dibujada fue resultado de un trabajo de articulación con la Asignatura Taller de Diseño Gráfico, I a partir de la cual se realizaron concursos entre los alumnos de primer año de la carrera de Diseño Gráfico, utilizándose posteriormente el afiche, los mailings y los certificados diseñados por el equipo ganador ${ }^{10}$. (Fig. 2).

[9 ] Esta actividad se articuló con las Cátedras de: Arq. III-UPC, Gestión y Desarrollo de la Vivienda Popular, Teoría del Diseño y la Gestión Urbana, Teoría y Método II e Historia y Crítica de la Arquitectura III. Ver ALCALA, L.; FOULKES, M. D.; PEREZ, R.; SCORNIK, M.; BRITO, C.; SOROCHIN, J. (2010). 
2. Cuadro síntesis de expositores y de temas. Elaboración propia

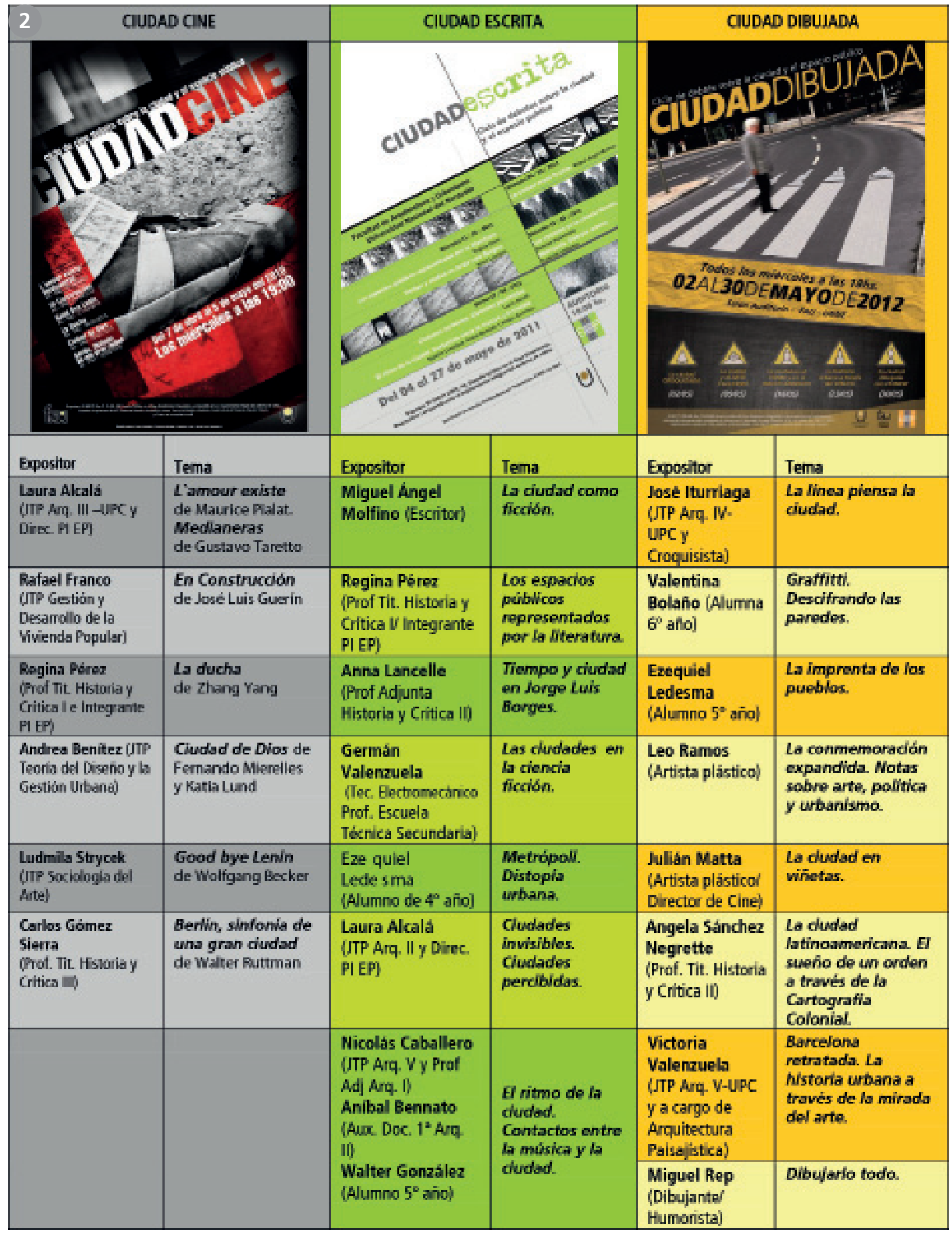




\section{RESULTADOS}

A continuación enumeraremos algunos resultados sobre los que posteriormente dejaremos abiertas algunas reflexiones.

1. Los Ciclos tuvieron una participación promedio de alrededor de cien personas, algunas jornadas tuvieron el doble de participación, mientras en otras el número fue ostensiblemente menor. Debe tenerse en cuenta que cada Ciclo se desarrolló en horario superpuesto al del taller y que los estudiantes no siempre obtenían el permiso de sus docentes para asistir, en unos casos porque los Responsables de Cátedra se negaban abiertamente a ello y en otros porque inevitablemente no podían suspenderse actividades programadas. En este sentido, es comprensible que el desarrollo del Ciclo impactaba en la programación de clases, al repetirse durante un mes el mismo día miércoles. Ello fue analizado varias veces por el equipo organizador, pero siempre se llegó a la conclusión de que era la alternativa más viable de desarrollo, teniendo en cuenta la mayor flexibilidad de los días de taller y la conveniencia de compactar la actividad en un mes, más allá del cual se debilitaría. 2. Entre los participantes, un dato significativo es que la mayor parte de ellos lo fue de los 3 Ciclos, y que entre ellos se verificó la participación voluntaria en el o los ciclos siguientes de quienes fueran participantes obligados al cursar Arquitectura III.

3. En cuanto a la participación de la comunidad no académica, fue muy reducida, circunscribiéndose fundamentalmente a los días en que participaron el escritor Miguel Ángel Molfino y el dibujante y humorista Miguel REP. También en el caso de la exposición de los alumnos asistieron padres y familiares. Un dato no menor es que también llegaron a participar estudiantes del colegio secundario.

4. Ninguna de las personas invitadas a ser expositores (ni docentes, ni estudiantes, ni artistas) se

[10 ] Al respecto puede leerse: ALCALÁ, L. y PELLI, B. (2010) negaron o rehusaron a serlo. Todas se mostraron interesadas y demostraron en sus exposiciones el tiempo y empeño dedicado a la preparación del tema propuesto, que en muchos casos obligó al desarrollo de un verdadero trabajo de investigación y de recolección de información. Las exposiciones se caracterizaron por la profundidad y calidad de contenidos, por la curada organización expositiva y por la riqueza del material gráfico incluido. Un dato significativo frente a esta dedicación es que se trató de un trabajo ad honorem, con excepción del expositor que debió desplazarse desde Bs. As. 5. Las instancias de debate no siempre llegaron a producirse realmente. Manifestaciones posteriores de los participantes de acercamiento y comentarios a los expositores o a los organizadores demostraron más bien que ello puede atribuirse no a la falta de interés sino a la timidez de hablar frente a un auditorio numeroso, a la dificultad de pasar de manera directa de una modalidad expositiva a la de debate abierto, también al cansancio natural que se verifica en la última parte de la jornada académica. En muchos casos también, la participación entusiasta de profesores asistentes restó espacio a la palabra de los estudiantes algoque la coordinación no siempre pudo manejar. Sin embargo, puede decirse que la mayor participación desinhibida de los estudiantes coincidió con la presencia de expositores externos a la comunidad universitaria, por ejemplo, con el escritor, con el docente del colegio secundario, con los artistas plásticos y con el dibujante.

6. Entre los temas que se trataron a lo largo de los tres ciclos podemos citar, a modo de ejemplo:

En el Ciclo Ciudad Cine: - el impacto de los nuevos sistemas de información y de las condiciones de vida en las grandes ciudades que propenden a la autoexclusión de las personas en los espacios privados, siendo sin embargo el EP donde vuelven a reencontrarse con la vida y consigo mismas; - el impacto de la modernidad en los espacios colectivos de los grandes conjuntos habitacionales, el cambio de costumbres, de usos y de espacios de 
Podemos afirmar que estos Ciclos, en tanto estrategias de borde didáctico, se consolidaron en el transcurso de las tres ediciones cobrando una identidad propia.

socialización frente al paso de la vivienda individual a la colectiva, - las características de la vida en el EP de las periferias en la infancia y en la ancianidad; -los efectos de la gentrificación producida por las operaciones de transformación en los cascos antiguos, la estrecha relación entre el cambio del paisaje urbano y de los usuarios del EP; - el cambio de estética del EP, de actitudes y conductas en el EP a partir del cambio de regímenes políticos; - el rol de determinados espacios colectivos en culturas milenarias y el papel del agua en la socialización que se produce en ellos, la exclusión de género en estos espacios, las prácticas cotidianas deportivas en el EP; -el rol del EP en la ciudad en la entrada a la modernidad, el papel de los medios masivos de transporte como el tren o el tranvía, los desplazamientos masivos coincidentes con la subdivisión y especialización del trabajo, las horas pico, las manifestaciones gremiales en los EP, las luces de neón en el nuevo paisaje urbano, etc.

En el Ciclo CIUDAD ESCRITA: - la ciudad natal desde el exilio, la memoria urbana selectiva de la infancia, la percepción de los cambios y permanencias en el EP y en la gente al regreso, la ciudad ficcionada que sin embargo mejor describe a la real; la ciudad tatuada de la democracia; - las utopías y distopías urbanas planteadas por la ciencia ficción, la realidad de determinadas ciudades que materializaron e incluso superaron a las de la ciencia ficción, la estratificación y segregación de clases y sus correlatos en las áreas y espacios segregados de la ciudad en altura o en horizontal, las áreas artificiales protegidas y encapsuladas y las miserias de quienes quedan excluidos de ellas; - la ciudad y sus lugares (los cafés, las esquinas, determinadas calles) que están impregnados de nuestras propias historias personales; la ciudad evocada en las letras de los tangos y en las poesías; -la noción del transcurrir del tiempo en la ciudad y en el EP; - las circunstancias, los sentimientos e historias personales que condicionan la percepción de las ciudades; - la ciudad natal a partir de la que uno percibe cualquier otra ciudad; los rasgos invariantes de cualquier ciudad y los cambiantes, etc.

En el Ciclo Ciudad Dibujada: -la captación intencional de un instante o de un lugar en un croquis urbano, la reflexión que lleva al dibujo de cada línea y la reflexión que queda abierta a partir de la línea dibujada; - los mensajes y expresiones escritos y dibujados en las calles, las tribus urbanas, las prácticas marginales en el EP, arte urbano o vandalismo; - las instalaciones e intervenciones con mensajes político-reivindicativos; -el arte urbano como herramienta para incidir en el pensamiento colectivo; -la ciudad de los superhéroes y de los antihéroes; la idea de acción y movimiento a través de las líneas del dibujo, del tiempo a través de la organización y tamaño de la escena y las viñetas, los colores que crean ambientes y estados anímicos; - los dibujos que intentan negar un territorio y crear una ciudad ideal ordenada, - el dibujo que incide en la manera de ver y entender una ciudad; -la posibilidad de reconstruir la historia de una ciudad, evocar sus condiciones de vida, las situaciones políticas, el progreso, el deterioro, a través de las pinturas que hicieron los artistas de ella; - la identidad de cada barrio expresada a través del dibujo, los edificios icónicos, las tramas singulares, los espacios marginales, las regularidades y las discontinuidades, las características físicas que permiten diferenciarlos o no de los otros barrios, etc.

\section{REFLEXIONES FINALES ABIERTAS}

Podemos afirmar que estos Ciclos, en tanto estrategias de borde didáctico, se consolidaron en el transcurso de las tres ediciones cobrando una identidad propia. Seguramente contribuyó a ello el tipo y características de la propuesta, distinta de las prácticas habituales de seminarios o encuentros con normas y comités de referato, pero también seguramente, el hecho formal de que, aun fuera de los parámetros curriculares tradicionales, se hayan planteado con una estructura y un formato propios, que se repitieron invariantemente los 
tres años, produciendo así la idea de continuidad y con ello la posibilidad de apropiación y de hábito entre quienes lo siguieron. Algunos elementos como: la reiteración de un tipo de denominación común (Ciudad Cine, Ciudad Escrita, Ciudad Dibujada); la repetición de un espacio y de una modalidad de desarrollo; un mismo día de la semana (los miércoles) durante el mismo mes de cada año (mayo) contribuyeron inevitablemente a cargarlo de esa identidad. Esta identidad y permanencia contribuyó también, seguramente, a ganarse una cuota de credibilidad o de respeto en el mismo ámbito universitario. Por tanto, podríamos pensar que los procesos de aprendizaje generados por estrategias de borde didáctico no necesariamente son prácticas marginales, pueden formar parte de propuestas sistemáticas complementarias y enriquecedoras de los espacios curriculares, por cierto absolutamente necesarios.

El problema radica en que no se da el entorno favorable que permita que, quienes tienen inquietudes transdisciplinarias, se encuentren. (MAX NEEF, 2003)

La posibilidad de haber sido sostenidos estos ciclos en el tiempo habla de distintas cosas, desde el apoyo institucional recibido para hacerlo (respaldo a partir de la declaración de interés en cada caso por parte del Consejo Directivo de la FAU, habilitación del espacio y apoyo técnico para su desarrollo, apoyo para la difusión); al compromiso y entusiasmo puesto por el equipo de investigación; a la favorable receptividad que tuvo la propuesta, a la generosa predisposición de los expositores, etc. De alguna manera ello expresa la predisposición y, por qué no, la necesidad de muchos de los integrantes de la comunidad académica de participar, de integrarse, de formar parte de espacios creativos e innovadores de construcción del conocimiento, aunque ello insuma un tiempo y un esfuerzo extras a sus labores habituales y no suponga, desde el punto de vista formal vigente, un rédito curricular significativo. En el plano docente, lejos de pretender asociar estos espacios a prácticas transdisciplinarias -aunque esta sea la utopía de referencia-, entendemos que hay en estas actitudes una búsqueda interna y una actitud abierta hacia espacios despojados de convencionalismos (relaciones jerárquicas normadas entre docentes y alumnos, fragmentación rígida de tipos de conocimientos en las distintas asignaturas), deseos permanentes de aprendizaje e inquietudes positivas hacia la construcción de un tipo de conocimiento en el que los límites disciplinarios se difuminen en pos de abordajes más holísticos e integrales. Este tipo de espacios extra-aúlicos podrían considerarse, entonces, catalizadores de estas sinergias positivas.

En relación con los estudiantes, merece tenerse en cuenta el seguimiento voluntario de los ciclos, el interés desarrollado por un tema que aparece marginado en la currícula de la Carrera y que sin embargo es sustantivo para quienes habrán de actuar, intervenir, transformar con sus proyectos y obras la ciudad, el sitio, el EP.

"Profe, el año que viene toca la música... nosotras los ayudamos". (Alumnas de $6 .^{\circ}$ año, asistentes de los 3 ciclos y alumnas de Arq. III UPC en el año 2009, anterior a los ciclos). Más allá del acercamiento propiciado a un tema que consideramos central, creemos que existe también una llamada de atención sobre cómo lograr producir la aproximación a un tema, la necesidad de innovar en formatos distintos de los que se reproducen en general dentro de las aulas.

La participación de estudiantes en tanto expositores en situación de igualdad con docentes y artistas, rompe no solo con las estructuras hegemónicas en las que se produce habitualmente el proceso de enseñanza-aprendizaje, sino también con el quién es el sujeto de aprendizaje. Al menos dentro del equipo de investigación, la percepción en todos los casos fue de aprendizaje permanente, tanto de nuestros pares expositores como de los estudiantes expositores, quienes fueron capaces de desarrollar narrativas y conceptos de similar nivel de profundidad e interés.

No puede soslayarse el hecho de que la propia invi- 
Se trata en definitiva de un modelo basado en la indagación, en el planteo de nuevas preguntas en contextos des acartonados, o fuera de las reglas convencionales curriculares. Y a partir de los descubrimientos individuales en esas búsquedas, fue posible construir un modelo de aprendizaje cooperativo. (LITWIN, 2011:90).

tación a un estudiante a desarrollar un tema en un rol de estas características supone un desafío especial y el desencadenamiento de dosis importantes de creatividad, interés y energías para dar lo mejor de sí. Este es otro aspecto significativo a la hora de tener en cuenta las capacidades latentes que poco contribuimos a desarrollar en las prácticas habituales de los procesos de enseñanza en los cuales los estudiantes tienen un rol absolutamente pasivo.

El efecto de la luz podrá ser un tema de la física, de la biología, de la astronomía, pero también podrá ser tema de la imaginación en las Catedrales de Rouen que Claude Monet pintó sucesivas veces con mágicas pinceladas, transformando esa Catedral al apreciar la luz y sus efectos sobre ella. (Edith LITWIN, 2011:19)

El uso del arte como herramienta didáctica demostró tanto el poder de convocatoria para trabajar sobre un tema problema como la ciudad y el espacio público (quizás como consecuencia en parte del placer estético que el arte siempre produce más allá del tema con él trabajado), como el poder ilustrativo y reflexivo para plantear ideas, problemas, contextos. El arte apela a los sentidos de un modo que el discurso científico no puede hacerlo, y desencadena estados emocionales y sentimientos que facilitan la comprensión de los contenidos teóricos, de la misma manera que nos muestra de manera flagrante las limitaciones disciplinares para explicar una realidad compleja y diversa.

El listado -que podría juzgarse caótico- de temas surgidos en los Ciclos es una clara muestra de lo que el arte nos indujo a pensar y problematizar sobre un mismo tema de estudio, muchos de los cuales difícilmente formen parte de los contenidos de los programas de las asignaturas o de estudios e investigaciones disciplinares y que, sin embargo, son parte de esa realidad compleja y diversa que pretendemos explicar y en muchos casos modificar. En otros casos, el poder expresivo y de síntesis del arte contribuye a clarificar de un modo admirablemente simple y asimilable nociones de extrema complejidad y dificultad comprensiva.

Este listado amplio de temas, y los matices y apre- ciaciones vertidos en torno a ellos, también es reflejo de la diversidad de expositores, de sus saberes y de sus posicionamientos político-ideológicos. La posibilidad de visibilizar las distintas perspectivas consideramos que constituye desde el punto de vista del aprendizaje una condición fundamental para aprender a relativizar la universalización de las propias ideas, el desarrollo de la capacidad de tolerancia, comprensión y respeto hacia las ideas distintas y la necesidad de construir aproximaciones integradoras sobre objetos de estudios como la ciudad o el espacio público que, por ser construcciones sociales, no admiten verdades únicas. Los Ciclos se basaron en la hipótesis de que determinadas asociaciones entre formas expresivas artísticas y temas vinculados con la ciudad y el espacio público podían desencadenar nuevos conocimientos o resignificar conocimientos sobre estos. En definitiva, los Ciclos se construyeron planteando preguntas o enunciados a los expositores que los obligaron de algún modo a investigar o indagar. Sus exposiciones fueron la transmisión de aquello que habían descubierto al hacerlo. En el caso de los artistas, supuso la reflexión sobre la propia obra, sobre el propio hacer.

Se trata en definitiva de un modelo basado en la indagación, en el planteo de nuevas preguntas en contextos des acartonados, o fuera de las reglas convencionales curriculares. Y a partir de los descubrimientos individuales en esas búsquedas, fue posible construir un modelo de aprendizaje cooperativo. (LITWIN, 2011:90).

Desde el punto de vista de la investigación que dio marco a esta experiencia, los resultados contribuyeron a la Fase 1, de construcción de marco teórico. Desde el punto de vista de la práctica docente en el taller de Arquitectura, el desarrollo en paralelo de los ciclos con la UT1, de diseño urbano de un sector de la ciudad, contribuyó a generar un bagaje de conocimientos y reflexiones que de otro modo no hubiera sido posible dentro del espacio del taller, pero en el que sí, en cambio, pudieron apreciarse procesos de asimilación de los contenidos de los Ciclos en los resultados de los trabajos 
prácticos producidos.

Por último, podemos decir que los Ciclos fueron una experiencia que permitió demostrar una, de las seguramente infinitas, formas posibles de articulación entre docencia e investigación y de cómo esta articulación concreta entre dos equipos de trabajo (de investigación y de docencia) puede propiciar a su vez, la apertura e integración con otros actores de la comunidad académica y externa a ella, enriqueciendo significativamente los resultados.

\section{BIBLIOGRAFÍA}

- Alcalá, Laura (2010). "Panorama del espacio público en el Gran Resistencia. Postales de las diferencias y de la indiferencia". Ponencia presentada al $1^{\circ}$ SEMINARIO LAS CALLES Y EL ESPACIO PÚBLICO. Pensamiento, diseño, gestión y realidad. Organizado por el PI SGCyT C001/08. y la FAU-UNNE, 23 y 24 de septiembre de 2010. Publicado en soporte electrónico (CD) ISBN 978-950-656-133-8.

- Alcalá, L.; Foulkes, M D.; Pérez, R.; Scornik, M.; Valdés, P.; Bennato, A.; Sorochin J. Y González, W. (2012). "Ciclo Ciudad Escrita. Una experiencia de articulación entre investigación y docencia". Pp. 211-214. En Comunicaciones Científicas y Tecnológicas Anuales 2011 EDIFAU. Facultad de Arquitectura y Urbanismo. UNNE. 2012. ISSN.1666-4035MAX.

- Alcalá, L.; Foulkes, M D.; Pérez, R.; Scornik, M; Brito C.; Sorochin J. (2010) "Ciclo Ciudad Cine. Construcción experimental de una comunidad de aprendizaje sobre la ciudad y el espacio publico". Pp. 227-230. En Comunicaciones Científicas y Tecnológicas Anuales 2010. EDIFAU. Facultad de Arquitectura y Urbanismo. UNNE. 2011. ISSN.1666-4035.

- Alcala, Laura Y Pelli, Ma. Bernabela (2010). "Imagen gráfica del Ciclo Ciudad Cine. Una experiencia de integración entre las Carreras de Arquitectura y Diseño Gráfico". Pp. 215-218. En Comunicaciones Científicas y Tecnológicas Anuales 2010. EDIFAU. Facultad de Arquitectura y Urbanismo. UNNE. 2011. ISSN.1666-4035.

- Alcalá, Laura, Foulkes, Ma. Dora Y Scornik, Marina (2009) "Experiencia de investigación y diseño en torno al tema del espacio público y el sistema de calles del Gran Resistencia" (Pág. 219-222) En Comunicaciones Científicas y Tecnológicas Anuales 2009. Facultad de Arquitectura y Urbanismo, UNNE. ISSN: 1666-4035.

- Alcalá, Laura, Foulkes, Ma. Dora Y Scornik, Marina (2009). "El trabajo sobre ejes urbanos en el Gran Resistencia como posibilidad de articulación de actividades de investigación, enseñanza y extensión". Ponencia. XXVIII Encuentro y XIII Congreso de Escuelas y Facultades Públicas de Arquitectura de la región del MERCOSUR: «La enseñanza de la arquitectura». Santa Fe. Facultad de Arquitectura, Diseño y Urbanismo de la Universidad Nacional del Litoral. 21, 22 y 23 de Octubre de 2009. ISBN 978-987-657-202-6.

- Flecha García, Ramón y Puigvert, Lídia. "Las comunidades de aprendizaje. Una apuesta por la igualdad educativa" Universidad de Barcelona, España. http://comunidadesdeaprendizaje.net/pdf/flecha_puigvert_02.pdf

- Freire, Paulo. (1993) Pedagogía de la esperanza. Un reencuentro con la pedagogía del oprimido. Editorial Siglo Veintiuno. México, 2013.

- Litwin, Edith (2011). El oficio de enseñar. Condiciones y contextos. Editorial PAIDÓS. Bs.As. 2011.

- Max Neef, Manfred (2003). "Transdisciplina, para pasar del saber al comprender". Conferencia en la Universidad de Antioquia. http://disi.unal. edu.co/ /ctorress/PSist/PenSis07.pdf 